\title{
Mini-Implantate und ihre Eignung für ältere Patienten
}

Egal, ob es sich um Interessierte, Einsteiger oder erfahrene Anwender von MiniImplantaten handelt - das MDI-Symposium „Mini-Implantate im Zeichen des demografischen Wandels“ hat für alle etwas zu bieten. Denn bei dem Event am 23. November 2013 im Sofitel Munich Bayerpost im Herzen Münchens erhalten sie aus 1 . Hand Informationen zur Therapieoption der Prothesenstabilisierung mit den Durchmesser-reduzierten Minis. Auf dem Programm stehen zahlreiche Vorträge, in denen Experten aus Wissenschaft und Praxis die besondere Eignung von MDI für die Behandlung älterer Patienten beleuchten.

In der 1. Session beschreibt Prof. Dr. R. Biffar (Universität Greifswald) die mit dem Wandel verbundenen Herausforderungen für die Zahnarztpraxis und hinterfragt, ob das aktuelle zahnärztliche Therapiespektrum den besonderen Anforderungen einer älter werdenden Patientenklientel gerecht wird. Unterschiedliche Möglichkeiten der Implantattherapie bei zahnlosen Patienten werden von Prof. Dr. J. Feine (McGill Universität
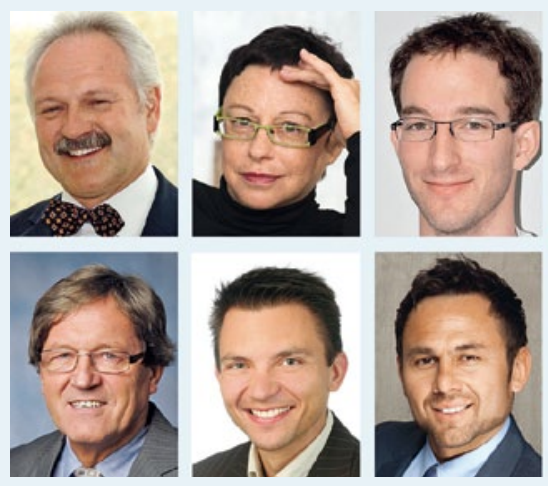

Montreal, Kanada) präsentiert, die in ihrem Vortrag die Sichtweise des Patienten in den Mittelpunkt stellt. Dr. A. Worni (Universität Bern, Schweiz) stellt aktuelle Studienergebnisse zum Einsatz von Mini-Implantaten bei der Verankerung von Totalprothesen vor, während PD Dr. F. Heinemann (Morsbach/Universität Greifswald) sich der Indikation der Pfeilervermehrung zur Verbesserung der Retention von Teilprothesen widmet.

Am Samstagnachmittag folgen fünf 20-minütige Kurzvorträge von niedergelassenen Zahnärzten aus Deutschland,
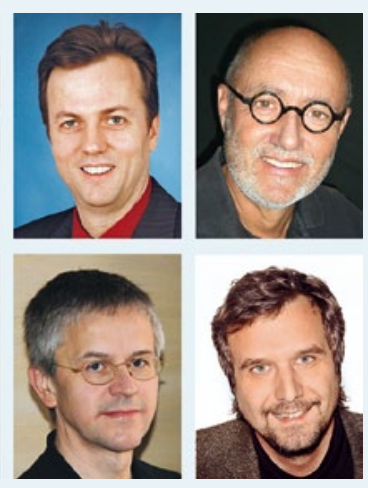

(oben v. I.) R. Biffar,

J. Feine, A. Worni und

F. Heinemann,

J. Hilgert;

(unten v. I.) W. Walzer,

Z. Keilinger, J. Z. Gal,

W. Tamminga,

T. Hauck.

die über langjährige Erfahrung mit Mini-Implantaten verfügen: Dr. J. Z. Gal (Ubstadt-Weiher), Dr. J. Hilgert (Drensteinfurt), Dr. Z. Keilinger (Schwäbisch Gmünd), Dr. W. Tamminga (Beckum) und Dr. W. Walzer (Berlin).

Für die Teilnahme am Symposium werden 8 Fortbildungspunkte nach den Leitsätzen der BZÄK und DGZMK vergeben. Die Teilnahmegebühr beträgt pro Person 299,-€ zzgl. MwSt. Das Kongressprogramm, weiterführende Informationen und Anmeldeformular finden Sie unter www.3MESPE.de/MDI-Symposium.

Nach einer Pressemitteilung der 3M Deutschland GmbH, Seefeld

\section{Mehr Behandlungsoptionen für mehr Flexibilität}

NobelReplace von Nobel Biocare ist ein weltweit häufig verwendetes 2-teiliges Implantatsystem. Einfach in der Handhabung und durch die prothetischen Komponenten nahezu für jede Indikation geeignet ist, steht es für vorhersehbare Ergebnisse. Gründe für Nobel Biocare kontinuierlich daran zu arbeiten, das System weiter $\mathrm{zu}$ entwickeln und die Produktlinie zu ergänzen.

Neues Mitglied in den Implantatfamilien NobelReplace Conical Connection und Replace Select Tapered ist ein Partially Machined Collar (PMC)-Implantat: Das neue Implantat mit einer Dreikanal-Innenverbindung hat eine 0,75-mm-maschinierte Schulter erhalten und bietet damit eine 2.Option zu dem bekannten Replace Select Tapered mit der 1,5-mmmaschinierten Schulter. Auch die Anwender der Produktlinie NobelReplace Conical Connection haben nun die Wahl zwischen dem Implantat mit struktu- rierter Implantatschulter mit TiUnite und der neuen PMC-Ausführung mit der 0,75-mm-maschinierten Schulter. Die Deckschraube ist bei beiden Implantaten sowie beim Replace Select Tapered im Preis enthalten.

Die Oberfläche der maschinierten Schulter zeigt eine Mikrorauigkeit, die der des natürlichen Zahnschmelzes (Ra 0,3-0,5) ähnelt. Dadurch wird die Anhaftung von Epithelzellen erreicht und die Weichgewebsanlagerung an die Implantatschulter und das Abutment unterstützt. Dies ermöglicht Anwendern den Anforderungen ihrer Patienten nach einer langfristig funktionellen und ästhetisch anspruchsvollen Versorgung gerecht zu werden.

Der Implantatkörper der gesamten NobelReplace-Familie bildet die Form einer natürlichen Zahnwurzel nach und ist unter den Aspekten einer optimalen Primärstabilität - bei allen Belastungsprotokollen einschließlich Sofortbelastung - und

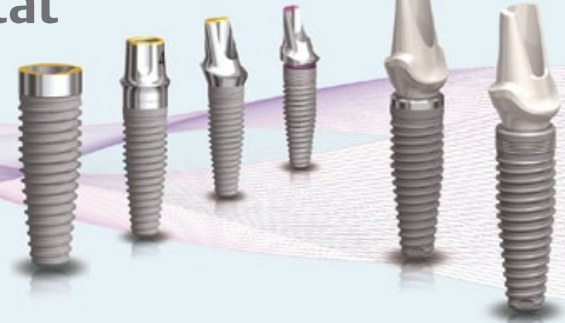

einer vorhersagbaren Osseointegration entwickelt worden. Unabhängig von Patientenbedürfnissen oder klinischen Präferenzen eignet sich das System für alle klinischen Anforderungen und mit seiner Auswahl an prothetischen Komponenten für nahezu alle Indikationen.

Die Replace-Familie bietet Einsteigern in die Implantologie eine solide Grundlage, auf der sie weiter aufbauen können. Erfahrene Anwender finden in diesem System ein zuverlässiges Allround-Implantat, das ihr gesamtes Behandlungsspektrum unterstützt.

Nach einer Pressemitteilung der Nobel Biocare Deutschland GmbH, Köln Internet: www.nobelbiocare.com 


\section{Schallzahnbürste als Black Edition}

Philips feiert den Erfolg der Philips Sonicare DiamondCleanSchallzahnbürste mit einer Black Edition. Wo sonst die Farbe Weiß vorherrscht, setzt die Black Edition neue Akzente.

Die iF-Juroren aus den Bereichen Material-, Verpackungs- und Produktkommunikationsdesign zeichneten 2012 die Schallzahnbürste mit dem iF communication design award aus. Aber nicht nur im Design, auch durch ihre Eigenschaft, souverän den Plaque-Biofilm zu managen, konnte die Bürste punkten - zuletzt im Vergleichstest (Test Heft 4/2013) der Stiftung Warentest. Dort erhielt sie für die Zahnreinigung ein „sehr gut“ $(1,4)$ und die Gesamtnote „gut“ $(1,6)$. Am 6.September wurde die Black Edition auf der Internationalen Funkausstellung in Berlin präsentiert, direkt danach ist sie auf den Fachdentals zu sehen. Die Philips

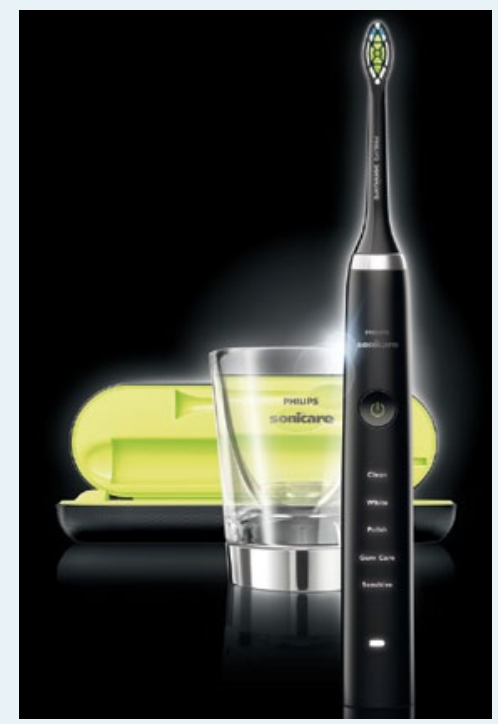

Sonicare DiamondClean Black Edition ist ab Oktober 2013 lieferbar.

Nach einer Pressemitteilung der Philips GmbH, Hamburg

Internet: www.philips.de/sonicare

\section{Partnerschaft geschlossen}

Die Unternehmen Zantomed GmbH (Duisburg) und Beewair (Macon Cedex, Frankreich) geben Ihre Partnerschaft für den Vertrieb der BeewairTechnologie in Deutschland und Österreich bekannt. Der französische Hersteller entwickelte unlängst eine neue, jetzt patentierte Luftdekontaminierungstechnologie namens DBD-Lyse ${ }^{\circledR}$, für die er bereits die Goldmedaille des französi-

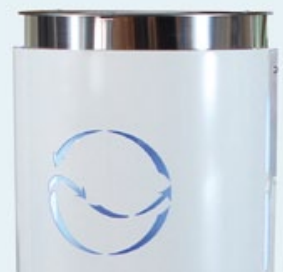

fung von keimfreieren Reinlufträumen im Rahmen chirurgischer Eingriffe, sondern trägt auch zu einem gesund erhaltenden Praxisklima bei. In Wartezimmern kann das Gerät die Patienten vor Viren und Bakterien schützen, die sonst $\mathrm{zu}$ einer möglichen Kreuzkontamination führen könnten. Gleiches gilt für nosokomiale Infektionen in Kliniken. In Behandlungsräumen werden schen Außenhandelsministeriums verliehen bekam.

Mit den Protect Air-Geräten ist es von nun an möglich auch Viren, Bakterien, Sporen, flüchtige Verbindungen und Allergene aus der Raumluft zu eliminieren und diese in ihren ursprünglichen - reinen Zustand zurückzuführen.

Dies ermöglicht nicht nur die Schafdank der neuen Technik auch flüchtige Verbindungen (Amalgam, Adhäsive, Lösungsmittel, Rückstände chemischer Desinfektionmittel etc.) aufgespalten, sodass diese durch Behandler, Praxisteam und Patienten nicht mehr aufgenommen werden. 